\title{
EEG-based endogenous online co-adaptive brain-computer interfaces: Strategy for success?
}

\author{
Reinhold Scherer \\ Institute of Neural Engineering \\ Graz University of Technology \\ Graz, Austria \\ Josef Faller and Paul Sajda \\ Laboratory for Intelligent Imaging \\ and Neural Computing \\ Columbia University \\ New York, NY, USA
}

reinhold.scherer@tugraz.at

\author{
Carmen Vidaurre \\ Dp. Mathematics \\ Public University of Navarre \\ Navarra, Spain and \\ Dp. Machine Learning \\ Berlin Institute of Technology \\ Berlin, Germany
}

carmen.vidaurre@unavarra.es

\begin{abstract}
A Brain-Computer Interface (BCI) translates patterns of brain signals such as the electroencephalogram (EEG) into messages for communication and control. In the case of endogenous systems the reliable detection of induced patterns is more challenging than the detection of the more stable and stereotypical evoked responses. In the former case specific mental activities such as motor imagery are used to encode different messages. In the latter case users have to attend sensory stimuli to evoke a characteristic response. Indeed, a large number of users who try to control endogenous BCIs do not reach sufficient level of accuracy. This fact is also known as BCI "inefficiency" or "illiteracy". In this paper we discuss and make some conjectures, based on our knowledge and experience in BCI, on whether or not online co-adaptation of human and machine can be the solution to overcome this challenge. We point out some ingredients that might be necessary for the system to be reliable and allow the users to attain sufficient control.
\end{abstract}

\section{INTRODUCTION}

A Brain-Computer Interface (BCI) translates patterns of brain signals such as the electroencephalogram (EEG) into messages for communication and control (for review see [1], [2], [3], [4]). To generate specific EEG patterns BCI users must perform the associated cognitive activities that elicit different mental states. The EEG is converted into patterns by means of feature extraction techniques (for a review see [3]) that improve the signal to noise ratio of the brain signals. To produce several mental states, the users either attend sensory stimuli that exogenously evoke specific patterns or endogenously induce patterns by mentally engaging in a task. These patterns are then translated into commands. In practice, the EEG information is not "just" visualized as done in neurofeedback, but is coded with the help of some algorithm, see Figure 1. Examples of exogenous signals are the P300 evoked potential [5], [6], [7], [8] or steady-state visual (or auditory or somatosensory) Evoked Potentials (EP) [9], [10]. The detection and translation of exogenous visual evoked responses, assuming that neural pathways are intact, can be achieved with accuracy of $90+\%$ and without learning curve over time [9]. Time of occurrence of the EP is linked to stimulus onset and is hence known. The shape of the

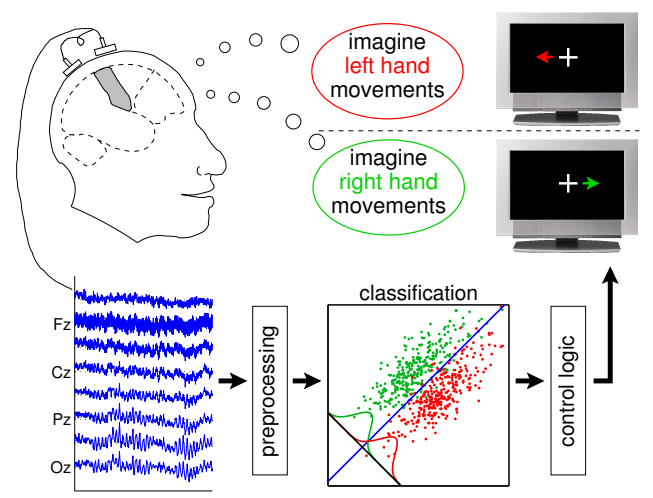

Fig. 1. Schema of a BCI system. The mental state of the user is acquired with some type of device. These signals are preprocessed to extract features and convert them into commands of, for example, a computer application. Thanks to the feedback, the user can observe the result of the interpretation of his/her mental state by the computer.

generated EP curve or other characteristic features of the EP are also known. Since these properties typically do not change significantly within an hour of time (the typical duration of $\mathrm{BCI}$ experiments in the laboratory) reliable translation of EP brain patterns into messages can be achieved. In end users with brain tissue damage that resulted in motor and cognitive impairment, the success rate strongly varies based on the related functional impairment (for example in end user with cerebral palsy [11]). High workload and other cognitive factors impact on EP features and change their properties over time, which leads to decrease in translation performance [12].

\section{The ROle of MACHINE LEARNING AND PATtern RECOGNITION}

The use of machine learning and pattern recognition significantly contributes to reduce training time and improve recognition performance [13], [14], [15], [16]. According to Tom Mitchell's definition a computer program is said to learn from experience, i.e. from data, with respect to some task and some performance measure, if its performance on 


\section{Calibration}

\section{Data acquisition with labels (information of mental states)}

\section{feature vectors \\ (training data)}

\section{feature space}

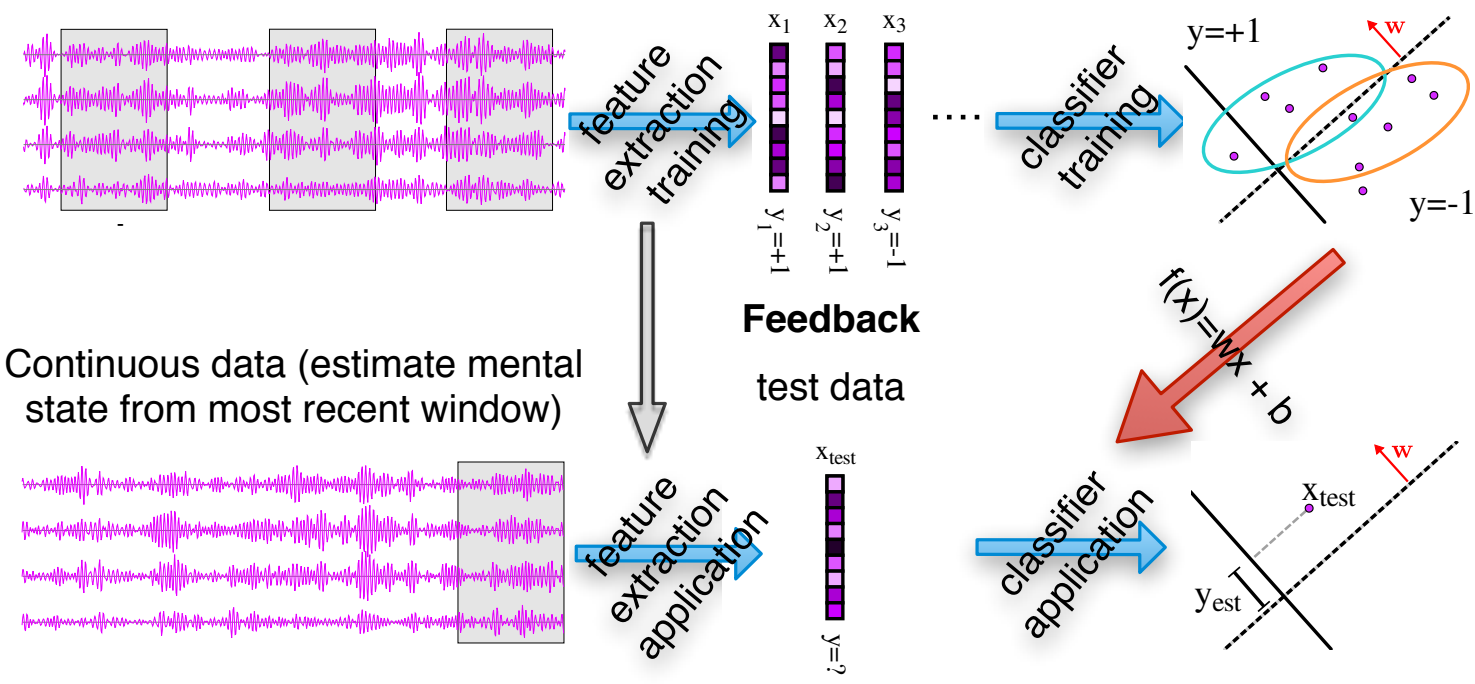

Fig. 2. Machine learning based BCI system. Usually a calibration session without feedback is performed to train the feature extraction and classifier methods. Just after that, the BCI session with feedback can start. The ongoing signal is classified according to what was learned in the calibration session.

the task, as measured by the performance metric, improves with experience [17]. Since we do not have comprehensive models describing the relationship between brain functioning, mental activities and EEG signals, data-driven approaches are used to characterize causal relationships. This means that we have to rely on experimental procedures for collecting data and statistical methods for describing signal features during different conditions (e.g. control vs. non-control). Statistical metrics such as the mean and the (co)variance are generally estimated from the collected data and used to characterize different experimental conditions (for example, the linear model of EP assumes that the mean of the signal contains the EP information and the (co)-variance the noise, [18]). Pattern recognition models are then selected based on the probability density function estimation of conditions and optimized to enhance translation accuracy. Linear methods often achieve high accuracy and reduce the system complexity (and are in line with the previously mentioned EEG model). Since EP properties are quasi-stationary and linear approaches achieve high performance, linear models are typically used for classification [19], [20], [18]. In this case it is typical that the ratio of the amount of training examples for target and nontarget class is highly unbalanced and this should be taken into account when parameters and performance are estimated [21], [18], [16]. Usually, a BCI session based on machine learning methods is divided into two parts. A calibration recording without feedback will serve to train the selected algorithms. As aforementioned, these typically include some feature extraction and classification technique. After that the feedback recording starts, in which the data is continuously classified. The already trained methods serve to estimate the current mental state of the user [22]. Figure 2 illustrates this 2-step approach.

\section{ENDOGENOUS BCIS}

The current paper focuses on BCIs that detect endogenously created patterns in the ongoing EEG. In this case specific mental activities are used to encode different messages. Performing such a specific mental activity induces transient changes in EEG and/or modulates EEG rhythms in a predictive way. Examples of mental tasks used to modulate oscillations in a predictive way are classic motor imagery tasks such as the repetitive kinesthetic imagination of hand or feet movements [23], attempted movement [24], or the solving of mental arithmetic or word generation tasks [25], [26]. Since the BCI user can start and stop performing the mental activity at will, endogenous BCIs intrinsically allow the establishment of userdriven communication (aka asynchronous or self-paced control [3], [27], [28], [29]). User-driven means that the user is in control and can initiate communication without the need of any exogenous stimulation or perturbation. While this is potentially useful it is at the expense of detection accuracy. The reason for accuracy deterioration lies in the nature of EEG. EEG is the summation of potential shifts that are generated by dendritic ion currents of millions of apical cortical neurons [30] [31]. Numerous short and long distance brain networks are active at any given time. Patterns resulting from the superposition of the activity of cortical neurons modulated by mental activities and the activity of short and long distance cortical networks may be subject to large variability both 
within and between subjects. This does not include technical or other sources of noise and interference that may contribute to EEG non-stationarity. Consequently, reliable detection of induced patterns is more challenging than the detection of the more stable and stereotypical evoked responses.

Experimental paradigms therefore often ask the user to modulate EEG oscillations in response to a cue. This reduces some uncertainty and limits analysis and interpretation of EEG to defined time intervals. Given the above-mentioned variability in inducing specific EEG patterns, and given the fact that we rely on statistical descriptors for characterizing variable pattern features and statistical pattern recognition techniques, the pattern recognition performance is lower compared to EP-based BCIs, but a learning curve can often be observed [25], [32], [33], [34]. Some BCI users are able to generate - in terms of statistical moments - very distinct patterns from the very beginning. Given that the distributions of features are different, machine learning is very successful in this group of users and pattern recognition performance is close to $100 \%$. The majority of users, however, is not able to reliably induce distinct patterns [35]. For discrimination between two different mental tasks accuracies in the range of $75 \%$ are commonly reported [35] [36]. Since BCI model parameters are typically fixated, users need to train to generate patterns that the machine can detect. Training requires feedback. Typically users perform mental activities and receive feedback on the pattern recognition performance. However, since patterns are not well established and hence not different enough, pattern recognition performance will be limited and the related feedback will include uncertainty. Unambiguous information makes learning and training very challenging for the user. Moreover, the related feedback EEG data may not be useful for improving translation performances since the human brain may start exploring different strategies, i.e. using various different mental activities, to be more successful. In summary, not only do we have to expect highly variable patterns, but also changes in EEG patterns due to the exploring of different mental activities that lead to reward, i.e., correct detection.

First training approaches used to gain BCI control were based on operant conditioning [37], [38]. This corresponds to the approach outlined above where BCI model parameters were fixated and the user had to train to modulate EEG oscillations by trial-and-error. Trial-and-error is the standard way how the brain learns. Humans try to perform a task, they get feedback on task performance and then they retry with adjusted parameters. Note, that this learning task is similar to the definition of machine learning. One major issue for BCI learning is the fact that the brain does not have a mental model of the BCI operation; nor sensors that measure the quality of cortical activity. Hence, the brain has to learn an internal representation of the $\mathrm{BCI}$ first, i.e., of the feature extraction and pattern recognition models. Once the BCI model is learned and internalized, which means that the brain gained sense of ownership and agency, it becomes an accepted extension of the body and control becomes more natural. This process, however, typically takes longer periods of time. Months and years of training are required. And this does not solve the issue of non-stationarity of EEG signals due to overlapping network activities discussed above. BCIs were developed with medical applications in mind. Years of training are not desirable. End users would need control as soon as possible to regain some level of independence.

Historically as next step machine learning was introduced in the BCI field. The directive was "let the machines learn". Indeed, sophisticated machine learning and pattern recognition methods were able to identify user-specific EEG pattern features within minutes or hours of time, depending on the type of algorithm used, and hence improve pattern recognition performance [39], [40]. However, keeping in mind the abovediscussed variability and non-stationarity properties of EEG patterns, this approach has obvious limitations.

In short summary: When only the brain is involved in the learning task (operant conditioning), then time plays a crucial role for achieving high performance. When learning is exclusively the task of the machine, then the intrinsic variability of EEG is a crucial factor for achieving high performance.

Nowadays, a common approach is to distribute the learning task and to co-adapt brain and machine [1], [41]. This means that machine learning and operant conditioning are applied alternately. Firstly, machine learning is applied to identify most discriminating EEG patterns in calibration recordings. Secondly, the brain trains to generate brain states by feedback training, aka. operant conditioning since BCI parameters are fixated. Thirdly, machine learning is again applied to the feedback data. Fourthly, feedback training is performed and so on. In this way machine and brain mutually co-adapt. This approach, however, corresponds basically to a connecting together of the two above limitations of each approach.

\section{IS ONLINE CO-ADAPTATION THE SOLUTION?}

The most natural way to overcome the aforementioned difficulties is to "let the machines learn" during the online operation of the system, that is, to apply machine learning methods during the ongoing experimental session. In this way, the machine explores the space of brain information and tries to minimize error or maximize information. Figure 3 summarizes online co-adaptation. This, of course, poses other difficulties: EEG is a noisy signal with low signal to noise ratio and the methods used should be robust against deviations that would not contain useful information [42], [43], [44]. Given this, one could think that not adapting might be then a safer way of reaching success (BCI control), because there might not be guarantee that the patterns learned online contain relevant information. Regarding this problem, a recent paper [45] showed with theoretical simulations of the 2-learners problem that the complete system, human and machine, converges in less time to a minimum error rate if both human and machine learn simultaneously from each-other. To reach this result one needs to take into account that the person should be able to correctly interpret the feedback given so that both, as a whole, try to minimize the committed mistakes. The 


\section{Online learning}

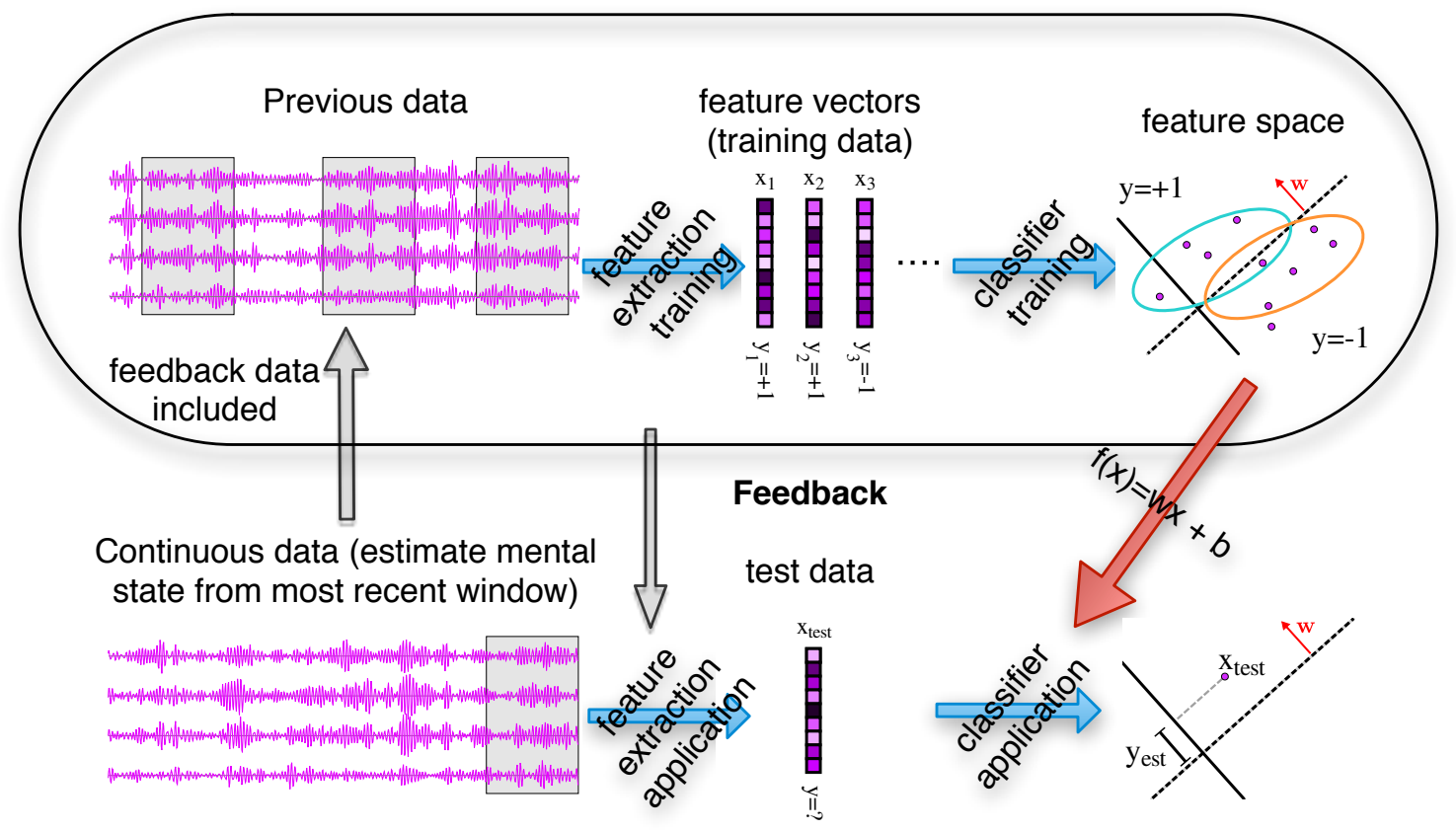

Fig. 3. The online co-adaptive machine learning-based BCI schema. Typically there is no need of a calibration session or this one might be very short. The data of the feedback session is used, maybe together with data of the same or other users, to train feature extraction and classification algorithms that will then be used to interpret the current mental state of the user.

authors of [45] also speculated that linear methods might deliver better results than non-linear ones because the user might better understand them, i.e., users are enabled to more quickly identify the relationship between mental activity and BCI output. Interpretability of feedback is one crucial issue that is believed to hamper success [46], [47], [48]. Online coadaptation and interpretability of the feedback in combination with ease of use were the basic reasons to develop a plug-andplay BCI [36]. The BCI collects a minimum number of trials (approx. 10 minutes of calibration data) that allows the machine learning algorithm to identify the one single band power feature that discriminates with high accuracy (Fisher Linear Discriminant Analysis classifier, LDA) between two mental states. After calibration the BCI switches automatically into the feedback training mode. Feedback training is performed with the selected individual feature. Feedback training thus corresponds to neurofeedback. The BCI analyzes the ongoing feedback EEG and recurrently updates the feature and LDA weights when an increase in performance is expected. In a supporting study, ten out of twelve novice users reached a criterion level of above $70 \%$ accuracy [49] in one to three sessions (10-80 min online time) of training, with a median accuracy of $80.2 \pm 11.3 \%$ in the last session. This performance was higher compared to the use of the conventional 2-stage training procedure described above. One advantage of such online learning systems is that the need of a calibration session is reduced [36], [50] or completely eliminated [51], [52], [53]. One typical option is to use data from other users to have an initial estimate of the necessary parameters [51], [52], [53]. Another common approach is to use previous data of the same user [54].

The state of the art of co-adaptive systems mostly shows successful outcomes for example with people who previously could attain sufficient BCI control [32], [55], [56], [33] or with patients [57]. Nevertheless there are also examples where co-adaptation did not work as good as expected. In [58], the classifier of a BCI system based on motor imagery was updated after every trial using context-aware guessed labels. The feature were extracted over three fixed laplacian locations (C3, Cz and C4) not optimized for the user. The frequency bands were also fixed. The authors reported that online adaptation was not useful for BCI training of users with poor performance. In [59] the authors used a fully automatic online adaptive BCI with completely naive users. The number of people who could not attain control was only slightly reduced in comparison to a semi-automatic machine learning based BCI. We speculate that in both cases the features did not contain relevant information. In the first case, the authors could not show that users with problems to attain control could improve performance with adaptation. However, these users have typically out of average EEG patterns [33], [59]. Due to this, the a priory selection of average laplacian locations and frequency bands does not seem, a priory, the most appropriate choice. Furthermore, from all the modules that compose a BCI system, only the classifier was updated. On the other hand in [59] the adaptation was performed in the location 
of the features and the classifier. After a number of runs Common Spatial Filters (CSF) were estimated and frequency bands updated. The selection of band and spatial filters was automatically performed. The authors speculate among other reasons that the selected features might have been unstable or prone to overfit for users with poor performance and that a BCI expert might help if this problems is timely detected. In any case, the authors of both papers hypothesize that online adaptation cannot overcome the selection of irrelevant features.

It should be noted that another factor that impacts on the overall system performance is user education and end user instruction. It is important to ensure that end users understand and are able to perform the required mental activities [60] [61]. If the BCI concept and mental task to be performed is not clear to the user, then online co-adaptation can not be successful.

\section{CONCLUSiON}

Endogenous BCIs systems are inefficient, when compared to exogenous BCIs, and a great number of users have problems to attain control ("BCI illiteracy" or "BCI inefficiency" [35]). The on-line coadaptation of the BCI system parameters seems to be promising to partly overcome problems encountered during the BCI operation [32], [55], [56], [33]. However, the methods applied should be robust against noise, simultaneously be able to explore the multivariate information space of the signals to extract relevant information. If the features do not contain discriminative information, co-adaptive systems will fail. Furthermore, the user needs to be able to understand the feedback [62] and it must probably be linearly linked to the relevant EEG features (power or amplitude) [45]. We conjecture that if all ingredients are properly designed, online adaptation offers the opportunity to readily include relevant new information to the feedback loop, thereby helping users to obtain BCI control.

\section{ACKNOWLEDGMENT}

C. Vidaurre was supported by grant number RyC-201415671 of the Spanish MINECO.

\section{REFERENCES}

[1] G. Pfurtscheller and C. Neuper, "Motor imagery and direct braincomputer communication," Proceedings of the IEEE, vol. 89, no. 7, 2001.

[2] J. R. Wolpaw, N. Birbaumer, D. J. McFarland, G. Pfurtscheller, and T. M. Vaughan, "Brain-computer interfaces for communication and control." Clinical Neurophysiology, vol. 113, no. 6, pp. 767-91, jun 2002.

[3] S. G. Mason, A. Bashashati, M. Fatourechi, K. F. Navarro, and G. E. Birch, "A comprehensive survey of brain interface technology designs." Annals of biomedical engineering, vol. 35, no. 2, pp. 137-69, feb 2007.

[4] J. D. R. Millán, R. Rupp, G. R. Müller-Putz, R. Murray-Smith, C. Giugliemma, M. Tangermann, C. Vidaurre, F. Cincotti, A. Kübler, R. Leeb, C. Neuper, K. R. Müller, and D. Mattia, "Combining BrainComputer Interfaces and Assistive Technologies: State-of-the-Art and Challenges." Frontiers in neuroscience, vol. 4, jan 2010.

[5] T. M. Vaughan, D. J. McFarland, G. Schalk, W. A. Sarnacki, D. J. Krusienski, E. W. Sellers, and J. R. Wolpaw, "The wadsworth BCI research and development program: At home with BCI," in IEEE Transactions on Neural Systems and Rehabilitation Engineering, vol. 14, 2006, pp. 229-233.
[6] C. Pokorny, D. S. Klobassa, G. Pichler, H. Erlbeck, R. G. L. Real, A. Kübler, D. Lesenfants, D. Habbal, Q. Noirhomme, M. Risetti, D. Mattia, and G. R. Müller-Putz, "The auditory P300-based singleswitch brain-computer interface: paradigm transition from healthy subjects to minimally conscious patients." Artificial intelligence in medicine, vol. 59, no. 2, pp. 81-90, oct 2013.

[7] E. W. Sellers, D. B. Ryan, and C. K. Hauser, "Noninvasive braincomputer interface enables communication after brainstem stroke," Science translational medicine, vol. 6, no. 257, p. 257re7, 2014.

[8] M. S. Treder, N. M. Schmidt, and B. Blankertz, "Gaze-independent brain-computer interfaces based on covert attention and feature attention," Journal of neural engineering, vol. 8, no. 6, p. 066003, 2011, open Access.

[9] B. Allison, T. Lüth, D. Valbuena, A. Teymourian, I. Volosyak, and A. Gräser, "BCI demographics: How many (and what kinds of) people can use an SSVEP BCI?" IEEE Transactions on Neural Systems and Rehabilitation Engineering, vol. 18, pp. 107-116, 2010.

[10] G. R. Müller-Putz, R. Scherer, C. Neuper, and G. Pfurtscheller, "Steadystate somatosensory evoked potentials: suitable brain signals for braincomputer interfaces?" IEEE Transactions on Neural Systems and Rehabilitation Engineering, vol. 14, no. 1, pp. 30-7, mar 2006.

[11] I. Daly, M. Billinger, J. Laparra-Hernández, F. Aloise, M. L. García, J. Faller, R. Scherer, and G. R. Müller-Putz, "On the control of braincomputer interfaces by users with cerebral palsy," Clinical Neurophysiology, vol. 124, no. 9, pp. 1787-1797, 2013.

[12] I. Käthner, S. C. Wriessnegger, G. R. Müller-Putz, A. Kübler, and S. Halder, "Effects of mental workload and fatigue on the P300, alpha and theta band power during operation of an ERP (P300) brain-computer interface," Biological Psychology, vol. 102, no. 1, pp. 118-129, oct 2014.

[13] B. Blankertz, G. Dornhege, C. Schäfer, R. Krepki, J. Kohlmorgen, K.-R. Müller, V. Kunzmann, F. Losch, and G. Curio, "Boosting bit rates and error detection for the classification of fast-paced motor commands based on single-trial EEG analysis," IEEE transactions on neural systems and rehabilitation engineering : a publication of the IEEE Engineering in Medicine and Biology Society, vol. 11, no. 2, pp. 127-131, 2003.

[14] P. Sajda, A. Gerson, K.-R. Müller, B. Blankertz, and L. Parra, "A data analysis competition to evaluate machine learning algorithms for use in brain-computer interfaces," IEEE transactions on neural systems and rehabilitation engineering : a publication of the IEEE Engineering in Medicine and Biology Society, vol. 11, no. 2, pp. 184-185, 2003.

[15] B. Blankertz, G. Dornhege, M. Krauledat, K.-R. Müller, V. Kunzmann, F. Losch, and G. Curio, "The Berlin Brain-Computer Interface: EEGbased communication without subject training," IEEE transactions on neural systems and rehabilitation engineering : a publication of the IEEE Engineering in Medicine and Biology Society, vol. 14, no. 2, pp. 147-152, 2006.

[16] S. Lemm, B. Blankertz, T. Dickhaus, and K.-R. Müller, "Introduction to machine learning for brain imaging," NeuroImage, vol. 56, pp. 387-399, 2011.

[17] T. M. Mitchell, Machine Learning. McGraw-Hill Science/Engineering/Math, 1997, vol. 4.

[18] B. Blankertz, S. Lemm, M. S. Treder, S. Haufe, and K.-R. Müller, "Single-trial analysis and classification of ERP components - a tutorial," NeuroImage, vol. 56, pp. 814-825, 2011.

[19] K.-R. Müller, C. W. Anderson, and G. E. Birch, "Linear and non-linear methods for brain-computer interfaces," IEEE transactions on neural systems and rehabilitation engineering : a publication of the IEEE Engineering in Medicine and Biology Society, vol. 11, no. 2, pp. 165169, 2003.

[20] F. Lotte, M. Congedo, A. Lécuyer, F. Lamarche, and B. Arnaldi, "A review of classification algorithms for EEG-based brain-computer interfaces." Journal of Neural Engineering, vol. 4, no. 2, pp. R1-R13, jun 2007.

[21] C. Vidaurre, N. Krämer, B. Blankertz, and A. Schlögl, "Time domain parameters as a feature for EEG-based brain computer interfaces," Neural Networks, vol. 22, pp. 1313-1319, 2009.

[22] B. Blankertz, G. Dornhege, M. Krauledat, K.-R. Müller, and G. Curio, "The non-invasive Berlin Brain-Computer Interface: Fast acquisition of effective performance in untrained subjects," NeuroImage, vol. 37, no. 2, pp. 539-550, 2007.

[23] C. Neuper, R. Scherer, M. Reiner, and G. Pfurtscheller, "Imagery of motor actions: differential effects of kinesthetic and visual-motor 
mode of imagery in single-trial EEG." Brain research. Cognitive brain research, vol. 25, no. 3, pp. 668-77, dec 2005.

[24] Y. Blokland, L. Spyrou, J. Lerou, J. Mourisse, G. Jan Scheffer, G.-J. van Geffen, J. Farquhar, and J. Bruhn, "Detection of attempted movement from the EEG during neuromuscular block: proof of principle study in awake volunteers," Scientific Reports, vol. 5, no. August, p. 12815, oct 2015.

[25] E. V. C. Friedrich, R. Scherer, and C. Neuper, "Long-term evaluation of a 4-class imagery-based brain-computer interface." Clinical Neurophysiology, vol. 124, no. 5, pp. 916-27, may 2013.

[26] R. Scherer, J. Faller, E. V. Friedrich, E. Opisso, U. Costa, A. Kübler, and G. R. Müller-Putz, "Individually adapted imagery improves braincomputer interface performance in end-users with disability." PloS one, vol. 10, no. 5, p. e0123727, jan 2015.

[27] J. d. R. Millán and J. Mouriño, "Asynchronous BCI and local neural classifiers: an overview of the Adaptive Brain Interface project." IEEE Transactions on Neural Systems and Rehabilitation Engineering, vol. 11, no. 2, pp. 159-61, jun 2003.

[28] R. Scherer, G. R. Müller, C. Neuper, B. Graimann, and G. Pfurtscheller, "An asynchronously controlled EEG-based virtual keyboard: improvement of the spelling rate." IEEE Transactions on Biomedical Engineering, vol. 51, no. 6, pp. 979-84, jun 2004.

[29] R. Scherer, F. Lee, A. Schlögl, R. Leeb, H. Bischof, and G. Pfurtscheller, "Toward self-paced brain-computer communication: navigation through virtual worlds." IEEE Transactions on Biomedical Engineering, vol. 55, no. 2 Pt 1, pp. 675-82, feb 2008.

[30] D. L. Schomer and F. L. D. Silva, Niedermeyer's Electroencephalography: Basic Principles, Clinical Applications, and Related Fields, 2010, vol. 1.

[31] R. Hari and L. Parkkonen, "The brain timewise: how timing shapes and supports brain function." Philosophical transactions of the Royal Society of London. Series B, Biological sciences, vol. 370, no. 1668, may 2015.

[32] C. Sannelli, C. Vidaurre, K.-R. Müller, and B. Blankertz, "Common spatial pattern patches - an optimized filter ensemble for adaptive braincomputer interfaces," Journal of neural engineering, vol. 8, no. 2, p. 025012 (7pp), 2011.

[33] _ _ "Ensembles of adaptive spatial filters increase bci performance: an online evaluation," Journal of neural engineering, vol. 13, no. 4, p. 046003, 2016

[34] C. Vidaurre and B. Blankertz, "Towards a cure for BCI illiteracy," Brain topography, vol. 23, pp. 194-198, 2010, open Access.

[35] B. Blankertz, C. Sannelli, S. Halder, E. M. Hammer, A. Kübler, K. R. Müller, G. Curio, and T. Dickhaus, "Neurophysiological predictor of SMR-based BCI performance," NeuroImage, vol. 51, pp. 1303-1309, 2010.

[36] J. Faller, C. Vidaurre, T. Solis-Escalante, C. Neuper, and R. Scherer, "Autocalibration and recurrent adaptation: Towards a plug and play online ERD-BCI," IEEE Transactions on Neural Systems and Rehabilitation Engineering, vol. 20, no. 3, pp. 313-319, 2012.

[37] N. Birbaumer, N. Ghanayim, T. Hinterberger, I. Iversen, B. Kotchoubey, A. Kübler, J. Perelmouter, E. Taub, and H. Flor, "A spelling device for the paralysed." Nature, vol. 398, no. 6725, pp. 297-8, mar 1999.

[38] A. Kübler, B. Kotchoubey, J. Kaiser, J. R. Wolpaw, and N. Birbaumer, "Brain-computer communication: unlocking the locked in." Psychological bulletin, vol. 127, pp. 358-375, 2001.

[39] B. Blankertz, R. Tomioka, S. Lemm, M. Kawanabe, and K. R. Müller, "Optimizing spatial filters for robust EEG single-trial analysis," IEEE Signal Processing Magazine, vol. 25, pp. 41-56, 2008.

[40] D. Steyrl, R. Scherer, J. Faller, and G. R. Müller-Putz, "Random forests in non-invasive sensorimotor rhythm brain-computer interfaces: a practical and convenient non-linear classifier." Biomedizinische Technik. Biomedical engineering, vol. 61, no. 1, pp. 77-86, feb 2016.

[41] R. Rao and R. Scherer, "Brain-Computer Interfacing [In the Spotlight," IEEE Signal Processing Magazine, vol. 27, no. 4, pp. 152-150, jul 2010.

[42] A. Schlögl, C. Vidaurre, and K.-R. Müller, "Adaptive methods in BCI research - an introductory tutorial," in Brain-Computer Interfaces, ser. The Frontiers Collection, B. Allison, B. Graimann, and G. Pfurtscheller, Eds. Springer, 2010, pp. 331-355.

[43] M. Kawanabe, W. Samek, K.-R. Müller, and C. Vidaurre, "Robust common spatial filters with a maxmin approach," Neural Computation, vol. 26, no. 2, pp. 1-28, 2014.

[44] C. Vidaurre, R. Scherer, R. Cabeza, A. Schlögl, and G. Pfurtscheller, "Study of discriminant analysis applied to motor imagery bipolar data," Med Bio Eng Comput, vol. 45, pp. 61-68, 2007.
[45] J. S. Müller, C. Vidaurre, M. Schreuder, F. C. Meinecke, P. von Bünau, and K.-R. Müller, "A mathematical model for the two-learners problem," Journal of Neural Engineering, vol. 14, no. 3, p. 036005, 2017.

[46] F. Lotte, F. Larrue, and C. Mühl, "Flaws in current human training protocols for spontaneous Brain-Computer Interfaces: lessons learned from instructional design," Frontiers in Human Neuroscience, vol. 7, p. 568, jan 2013.

[47] C. Jeunet, E. Jahanpour, and F. Lotte, "Why standard brain-computer interface (BCI) training protocols should be changed: an experimental study." Journal of neural engineering, vol. 13, no. 3, p. 036024, may 2016.

[48] R. Chavarriaga, M. Fried-Oken, S. Kleih, F. Lotte, and R. Scherer, "Heading for new shores! Overcoming pitfalls in BCI design," BrainComputer Interfaces, pp. 1-14, dec 2016.

[49] A. Kübler, N. Neumann, B. Wilhelm, T. Hinterberger, and N. Birbaumer, "Predictability of brain-computer communication," Journal of Psychophysiology, vol. 18, no. 2-3, pp. 121-129, 2004.

[50] A. Schwarz, R. Scherer, D. Steyrl, J. Faller, and G. R. Müller-Putz, "A Co-Adaptive Sensory Motor Rhythms Brain-Computer Interface Based on Common Spatial Patterns and Random Forest," in 2015 37th Annual International Conference of the IEEE Engineering in Medicine and Biology Society (EMBC), 2015, pp. 1049-1052.

[51] C. Vidaurre, A. Schlögl, R. Scherer, R. Cabeza, and G. Pfurtscheller, "A fully on-line adaptive BCI," IEEE Trans. on Biomed. Eng., vol. 53, pp. 1214-1219, 2006

[52] 1 , "Study of on-line adaptive discriminant analysis for EEG-based brain computer interfaces," IEEE Trans. on Biomed. Eng., vol. 54, pp. 550-556, 2007.

[53] R. Kobler and R. Scherer, "Restricted Boltzmann Machines in Sensory Motor Rhythm Brain-Computer Interfacing: A Study on Inter-Subject Transfer and Co-Adaptation," in 2016 IEEE International Conference on Systems, Man and Cybernetics SMC 2016, 2016.

[54] M. Krauledat, M. Tangermann, B. Blankertz, and K.-R. Müller, "Towards zero training for brain-computer interfacing," PloS one, vol. 3, no. 8, p. e2967, Aug 2008, open Access.

[55] C. Vidaurre, C. Sannelli, K.-R. Müller, and B. Blankertz, "Machinelearning based co-adaptive calibration," Neural computation, vol. 23, no. 3, pp. 791-816, 2011.

[56] — "Co-adaptive calibration to improve BCI efficiency," Journal of neural engineering, vol. 8, no. 2, p. 025009 (8pp), 2011.

[57] R. Scherer, J. Faller, E. Opisso, U. Costa, D. Steyrl, and G. R. MüllerPutz, "Bring mental activity into action! Self-tuning brain-computer interfaces," in 2015 Annual International Conference of the IEEE Engineering in Medicine and Biology Society, EMBC'15, 2015, pp. 2323 $-2326$.

[58] S. Perdikis, R. Leeb, and J. d R Millán, "Context-aware adaptive spelling in motor imagery bci," Journal of Neural Engineering, vol. 13, no. 3, p. 036018, 2016.

[59] L. Acqualagna, L. Botrel, C. Vidaurre, A. Kübler, and B. Blankertz, "Large-scale assessment of a fully automatic co-adaptive motor imagerybased brain computer interface," PloS one, vol. 11, no. 2, pp. 1-19, 02 2016, open Access.

[60] R. Scherer, A. Schwarz, G. R. Müller-Putz, V. Pammer-Schindler, and M. Lloria Garcia, "Game-based BCI training: Interactive design for individuals with cerebral palsy," in 2015 IEEE International Conference on Systems, Man and Cybernetics SMC 2015, 2015, pp. 3175-3180.

[61] — "Lets play Tic-Tac-Toe: A Brain-Computer Interface case study in cerebral palsy," in 2016 IEEE International Conference on Systems, Man and Cybernetics SMC 2016, 2016.

[62] R. Lorenz, J. Pascual, B. Blankertz, and C. Vidaurre, "Towards a holistic assessment of the user experience with hybrid BCIs," Journal of neural engineering, vol. 11, no. 3, p. 035007, 2014. 